\title{
EVOLUTION OF GALAXIES WITH PERIODIC STAR FORMATION
}

\author{
Satoru IKEUCHI \\ Tokyo Astronomical Observatory, \\ University of Tokyo \\ Mitaka, Tokyo 181, Japan
}

\begin{abstract}
Interchange processes in the interstellar medium, which is composed of diffuse gas, small clouds and giant molecular clouds are examined. It is stressed that the formation of giant molecular clouds through gravitational instability of a cloud ensemble leads to periodic star formation. The evolution of galaxies with such periodic star formation episodes is studied, especially paying attention to the effect of gas infall.
\end{abstract}

\section{FUNDAMENTAL PROCESSES OF STAR FORMATION IN THE ISM}

To study the evolution of galaxies, we must know the star formation rate (SFR). Till now it is usually assumed to be a power law function of the gaseous density, $\mathrm{SFR}=\alpha \rho_{\mathrm{g}}{ }^{\mathrm{k}}$. To see the physical foundations of this relation we must start the investigation from the fundamental physical processes for star formation in the interstellar medium (ISM).

We divide the ISM into three components: an ionized diffuse gas ( subscript $d$ ) composed of hot $\left(\sim 10^{6} \mathrm{~K}\right)$ and warm $\left(\sim 10^{4} \mathrm{~K}\right)$ gases, H I gas as small clouds ( subscript sc) with masses about $50 M_{\odot}$ and giant molecular clouds (subscript mc) from which stars are born.

The interchange processes among these components are simplified to the following three processes (Habe et al. 1981) :

(1) The diffuse interstellar gas is swept to cold dense shells by supernova remnants, and these dense shells are converted into small clouds by some hydrodynamical and/or gravitational instabilities. By this process the ionized gas is added to the cold component: the timescale of this process will be the time it takes supernova remnants to sweep the whole volume of a galaxy. This is of the order of $10^{7}$ years if we assume the standard value for the supernova explosion rate.

(2) For the growth processes of H I clouds to giant molecular clouds we 691

M. Peimbert and J. Jugaku (eds.), Star Forming Regions, 691-699.

(C) 1987 by the IAU. 
take two processes into account, mutual collisions of clouds with a timescale $\tau_{c c} \sim(5-10) \times 10^{7}$ years and gravitational instabilities of a cloud ensemble with a free fall timescale of the system, $\tau_{f f} \sim(3-5) \times 10^{7}$ years. This instability is assumed to occur if the cloud system in a differentially rotating disk satisfies the Toomre-Goldreich-Lynden Bell condition, that is, the surface density of a cloud system, $\Sigma_{s c}$, exceeds the critical one

$\Sigma_{\mathrm{sc}} \geq \Sigma_{\mathrm{crit}}=\gamma \frac{\kappa v}{\pi \mathrm{G}}$

where $\gamma$ is a numerical factor of order unity, $\kappa$ is the epicyclic frequency, $v$ is the velocity dispersion of the clouds and $G$ is the gravitational constant.

(3) The third is the destruction process of giant molecular clouds (GMCs) due to collapse to stars, ionization and evaporation by newly born stars and supernova explosions, and fragmentation to clumps. The timescale of destruction would be of the order of the travelling time over the cloud with isothermal sound speed, $\tau_{\mathrm{e}} \sim(2-3) \times 10^{7}$ years.

The above three procesises work coherently with a scale length of several kiloparsecs during several times $10^{7}$ years. This picture resembles that of the superclouds indicated by Elmegreen and Elmegreen (1983). Hereafter, we use the physical quantities averaged over this length scale.

\section{TWO BRANCHES OF STAR FORMATION IN A DISK GALAXY}

Since we suppose that stars are mainly born from giant molecular clouds, we examine first the time variation of the surface density of GMCs. The time variation of the surface density of GMCs, $\Sigma_{m c}$, is described as

$$
\frac{d}{d t} \Sigma_{m c}=-\frac{\Sigma_{m c}}{\tau_{e}}+\left(\frac{1}{\tau_{c c}}+\frac{\triangle(Q)}{\tau_{f f}}\right) \Sigma_{s c}
$$

where the step function $\triangle(Q)$ is defined as $\triangle(Q)=1$ for $Q>1$ and $=0$ for $Q<1$ with $Q=\Sigma_{\text {sc }} / \Sigma_{\text {crit }}$.

Here, it should be noted that the cloud collision time and free fall time depend upon the surface density of small clouds as

$$
\tau_{\mathrm{cc}} \propto \Sigma_{\mathrm{sc}}^{-1} \quad \text { and } \quad \tau_{\mathrm{ff}} \propto \Sigma_{\mathrm{sc}}^{-1 / 2}
$$

while the destruction time of GMC is independent of cloud abundance, $\tau_{\mathbf{e}}=$ const. 
Before going to the numerical integration of Equation (2) as well as those of the diffuse gas and H I clouds, we examine the steady state for GMCs. From this we can see the physical foundations of star formation processes. The steady state surface density of $\mathrm{H}$ I clouds and GMCs are, respectively, written as

$$
\Sigma_{s c}=\Sigma_{g}\left(\tau_{c c} \cdot \tau_{f f}\right) /\left(\tau_{c c} \cdot \tau_{f f}+\tau_{f f} \cdot \tau_{e}+\tau_{e} \cdot \tau_{c c} \Delta\right) \text { and } \Sigma_{m c}=\Sigma_{g}-\Sigma_{s c}
$$

where $\Sigma_{g}$ is the total surface density of clouds. From these we can expect two branches of star formation. One is named Branch $\mathrm{C}$, in which GMCs are formed by cloud-cloud collisions, because the gravitational instability condition is not satisfied. The other is named Branch I, in which GMCs are formed mainly by the gravitational instability in the cloud system.

The steady state aboundances and their dependence upon the total surface density $\Sigma_{g}$ are summarized as follows:

Branch $\mathrm{C}(\triangle(Q)=0)$

$\begin{array}{lcc} & \text { Low } \Sigma_{g} & \text { High } \Sigma_{g} \\ \Sigma_{\mathrm{sc}}^{c}=\frac{\tau_{\mathrm{cc}}}{\tau_{\mathrm{cc}}+\tau_{\mathrm{e}}} \sum_{\mathrm{g}} & \Sigma_{g} & \text { const. } \\ \Sigma_{\mathrm{mc}}^{\mathrm{c}}=\frac{\tau_{\mathrm{e}}}{\tau_{\mathrm{cc}}+\tau_{\mathrm{e}}} \sum_{g} & \Sigma_{g}^{2} & \Sigma_{g}\end{array}$

Branch I $(\triangle(Q)=1)$

Low $\Sigma_{g} \quad$ High $\Sigma_{g}$

$\begin{array}{lll}\sum_{s c}^{l}=\frac{\tau_{f f}}{\tau_{f f}+\tau_{e}} \sum_{g} & \Sigma_{g} & \sum_{g}^{1 / 2} \\ \sum_{m c}^{l}=\frac{\tau_{e}}{\tau_{f f}+\tau_{e}} \Sigma_{g} & \Sigma_{g}^{3 / 2} & \Sigma_{g}\end{array}$

As can be seen, the steady state surface density shows a weak dependence upon $\Sigma_{g}$. Therefore, when we take the star formation efficiency as $f_{S F}$ 
the star formation rate in each branch is written as follows :

Low $\Sigma_{g} \quad$ High $\Sigma_{g}$

$$
\begin{array}{lll}
\alpha_{\mathrm{SF}}^{\mathrm{c}}=f_{\mathrm{SF}} \frac{\sum_{\mathrm{mc}}^{\mathrm{c}}}{\tau_{\mathrm{e}}} & \Sigma_{g}^{2} & \Sigma_{g} \\
\alpha_{\mathrm{SF}}{ }^{1}=f_{\mathrm{SF}} \frac{\sum_{\mathrm{mc}}^{1}}{\tau_{\mathrm{e}}} & \Sigma_{\mathrm{g}}^{3 / 2} & \Sigma_{\mathrm{g}}
\end{array}
$$

In Figure 1, the star formation rates $\alpha_{\mathrm{SF}}{ }^{\mathrm{c}}$ and $\alpha_{\mathrm{SF}}{ }^{\mathrm{I}}$ are illustrated by dashed and solid lines, respectively, for two cases: $f_{\mathrm{SF}}=0.3$ and 0.025. The observationally estimated values for the SFRs are also plotted, and they are well fitted by the high efficiency case of Branch $\mathrm{C}$ and low efficiency case of Branch I. As for the critical density for Branch I the value at the solar neighborhood is assumed.

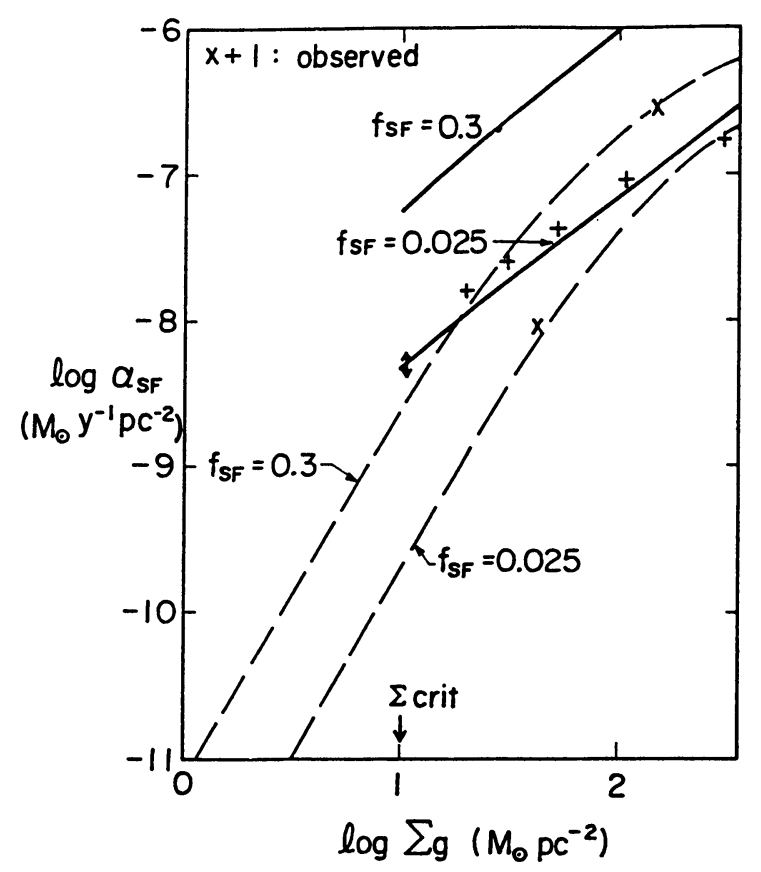

Figure 1. The Branch $\mathrm{C}$ star formation rate is represented by dashed lines and the Branch I by solid lines. Two cases of star formation efficiency are shown.

From the above result it is concluded that there is a big jump in the SFR at $\Sigma_{g} \sim \Sigma_{c r i t}$, and the dependency of the SFR upon $\Sigma_{g}$ becomes SFR $\sim \Sigma_{g}^{2}$ at $\Sigma_{g}<\Sigma_{\text {crit }}$ and $\sim \Sigma_{g}$ at $\Sigma_{g}>\Sigma_{\text {crit }}$.

\section{EVOLUTION OF GALAXIES}

Calculating the interchange processes among the diffuse gas, 
H I clouds and molecular clouds, we can obtain the time variations of the masses of the three components of the ISM as well as the stellar mass. Considering stellar data such as: lifetime, mass loss rate, final destination, metal abundance of ejected matter, color change and so on, we can follow the evolution of galaxies. Here, we present the time variations of the mass density of each gas component and of the stars, $\Sigma_{\text {st }}$. Two cases with and without gas infall are described separately.

\subsection{Case without gas infall}

In this case the evolution of galaxies is very simple. Depending upon the initial gaseous content, star formation begins at either Branch $\mathrm{I}$ or Branch $\mathrm{C}$, and the star formation rate as a whole decreases monotonically because of the gas consumption to stars. However, it should be noted that when the total gas density decreases nearly to the critical one we may expect a periodic evolution (Ikeuchi et al. 1984 ). Suppose that the total gas surface density is a little higher than $\Sigma_{c r i t}$ but the surface density of $\mathrm{HI}$ clouds is smaller than $\Sigma_{\text {crit. }}$ In this case, the star formation occurs at Branch C. Meanwhile, almost all the gas is converted to $\mathrm{H} \mathrm{I}$ clouds, and they exceed $\Sigma_{c r i t}$ As a result the star formation occurs at Branch I. Before long, the surface density of $H$ I couds becomes less than $\Sigma_{\text {crit. }}$ In this way, the star formation proceeds periodically changing between two branches and continues till the total gaseous density becomes smaller than $\Sigma_{\text {cirt }}$. A typical example of such a periodic evolution is shown in Figure 2, where $\Sigma_{\mathrm{sc}}, \Sigma_{\mathrm{mc}}$, and the supernova

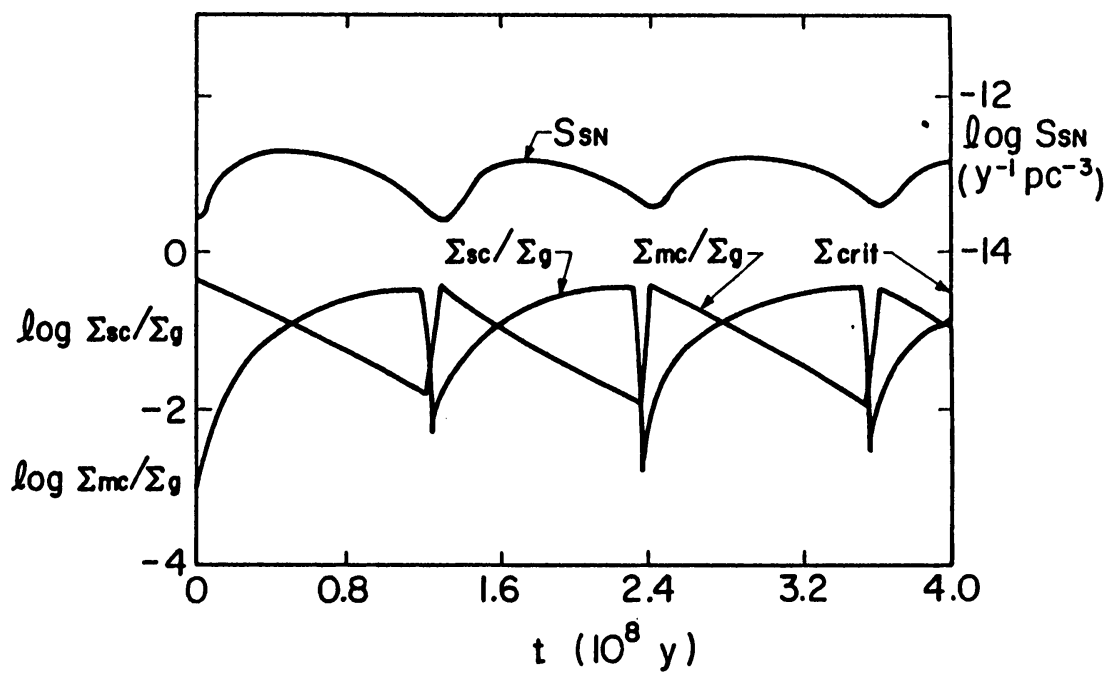

Figure 2. Time variations of $\Sigma_{s c}, \Sigma_{m c}$, and $S_{S N}$ for the case without gas infall. The gravitational instability occurs periodically. 
explosion rate $S_{\mathrm{SN}}$ are illustrated for the case of the solar neighborhood. The amplitude and period of star formation bursts depend upon $\kappa, \Sigma_{g}$ and $S_{S N}$. It should be noted that this periodic star formation is originated not in the dynamical process but in the physical interchange process. Namely, we may say the ISM has a proper rhythm. This periodicity ends when $\Sigma_{g}<\Sigma_{\text {cirt }}$, and then star formation will always occur at Branch $\mathrm{C}$.

\subsection{Case with gas infall}

Here, we assume that the gas infall rate has the following form

$$
\alpha_{\text {inf }}=\alpha_{0} \exp \left(-t / t_{\text {inf }}\right), \quad \sum_{\text {tot }}=\int_{0}^{t_{0}} \alpha_{\text {inf }} \mathrm{dt}
$$

where $\Sigma_{\text {tot }}$ is the total surface density in a disk and $t_{0}$ is the present age of a galaxy. We suppose that the gas is supplied as H I gas and its timescale $t_{\text {inf }}$ is a parameter.

The gas infall makes the gas surface density to increase, while the star formation makes it to decrease. Two competitive processes bring another periodicity to the star formation episode of a disk galaxy. In this case, as shown in Figure 3, the period of an oscillation is $\sim 10^{9}$ years and the amplitude of the change in the SFR is about a factor of 5, if we take the parameters for the solar neighborhood. Such a periodic star formation is surely expected when the gas infall rate is higher than the gas consumption rate at Branch $\mathrm{C}$ but is lower than that

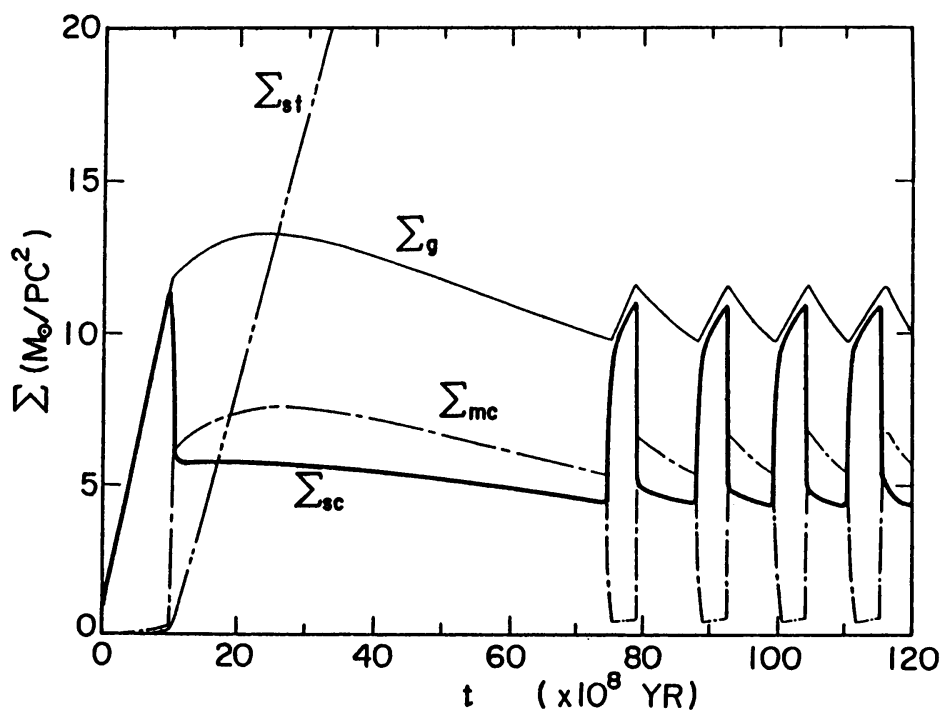

Figure 3. Evolution of a galaxy with periodic star formation episodes. Time variations of $\Sigma_{\mathrm{sc}}, \Sigma_{\mathrm{mc}}$, and $\Sigma_{\mathrm{st}}$ are shown. 
at Branch I. In this periodic model, since the star formation occurs intermittently between Branch I and Branch $C$ we name this type of solutions as Type (IC $)^{n}(n>1)$.

If the gas infall rate is always higher (lower) than the gas consumption rate at Branch I (Branch C), we name this type of solutions as Type I (Type C). The SFR of Type I solution is more than ten times higher than that of Type C solution.

\section{IMPLICATIONS OF THE PRESENT RESULTS}

\subsection{Correspondence of solutions to actual galaxies}

Type C : There are two kinds of Type C solutions. One is that the SFR is always small and this corresponds to the outside part of the Holmberg radius, at which the optical disk is not recognized clearly. The other is that the star formation was active at Branch I in the initial stage because of rapid gas infall, but almost all the gas was already consumed and the star formation is inactive at Branch $\mathrm{C}$ at present. Early $\mathrm{S}$ galaxies may correspond to this case.

Type $(\mathrm{IC})^{n}$ : This solution indicates the possibility of intermittent star formation episodes with a timescale of $\sim 10^{9}$ years. Such ( intermittent) star bursts indicated in Magellanic-type irregular galaxies may correspond to this Type (IC $)^{n}$ solution.

Type I : Since the star formation is very efficient even now this solution may correspond to the $4 \mathrm{kpc}$ molecular ring in our Galaxy and to the active galactic nuclei of $S$ galaxies.

\subsection{Other causes of periodic star formation}

We can imagine some other causes of the periodic star formation. They may be : (a) Dynamical expansion of gas, after the burst of star formation due to the rapid energy ejection, makes the star formation inactive. After that the gas released from evolved stars accumulates, and the second burst of star formation occurs. In this way, the burst-like star formation, the dynamical expansion of gas, the inactive star formation, the gas accumulation and the next burst of star formation occur successively. This model was originally presented by Loose et al. (1982). (b) Scalo and Struck-Marcell (1986) examined nonlinear behaviours of a cloud fluid model and found a limit cycle solution for the change of a cloud mass. They connected this solution with star formation bursts in galaxies. A similar idea of a cyclic phase change of the interstellar medium was already proposed by Ikeuchi and Tomita (1983), and this model was applied to the formation of dissipative structures in a differentially rotating disk by Nozakura and Ikeuchi (1984).

This work is based upon the results by Tanaka et al. (1985). 
Elmegreen, B.G., and Elmegreen, D.M.: 1983, Monthly Notices Roy. Astron. Soc., 203, 31 .

Habe, A., Ikeuchi, S., and Tanaka, Y. D.: 1981, Publ. Astron. Soc. Japan, 33, 23.

Ikeuchi, S., Habe, A., and Tanaka, Y. D.: 1984, Monthly Notices Roy. Astron. Soc., 207, 909.

Ikeuchi, S., and Tomita, H.: 1983, Publ. Astron. Soc. Japan, 35, 77.

Loose, H.H., Krugel, E., and Tutukov, A.: 1982, Astron. Astrophys., 105 , 342 .

Nozakura, T., and Ikeuchi, S.: 1984, Astrophys. J., 279, 40.

Scalo, J.M., and Struck-Marcell, C.: 1986, Astrophys. J., 301, 000.

Tanaka, Y.D., Ikeuchi, S., and Habe, A.: 1985, Sumbitted to Monthly Notices Roy. Astron. Soc., in April.

\section{DISCUSSION}

McKee : What are the implications of your model for star formation in galaxies with spiral density waves? Elmegreen has presented an evidence that the star formation rate per unit mass is the same in and between arms, yet the surface density is quite different in the two cases.

Ikeuchi : Elmegreen's result implies that the star formation rate $\alpha_{S F}$ is proportional to the gas surface density, and this corresponds to the star formation at Branch I with $\Sigma_{g}>\Sigma_{c r i t}$ for in and between arms. Therefore, there is no fundamental change of star formation process between them. Here, I would like to stress that the periodicity of star formaton rate in the present model comes from the physical interchange processes, not from the dynamical time. Therefore, if this periodicity coincides with the dynamical time like the period of the density wave the efficient star formation case may be expected.

Solomon : Our observation of GMC sizes and masses seem to indicate that both cloud collisions and gravitational instabilities are required to explain the small and large ends of the distribution. Your model appears to be conducted in combining both approaches. I have a question regarding the oscillations. You predict $\Sigma_{H I}$ to rise to high values yet observations of external galaxies indicate that $\Sigma_{\mathrm{HI}}$ seldom $\mathrm{rises}$ about $5 \mathrm{M}_{\odot} \mathrm{pc}^{-2}$.

Ikeuchi : Our model corresponds to a region with a length $\sim 2 \mathrm{kpc}$, and the numerical values should be averaged over the galaxy if we compare it with external galaxies. Then, the oscillation may be washed out, and the average $\Sigma_{H I}$ decreases to a smaller value. 
Pudritz: Have you considered the loss of hot phase in superbubbles out of the disk plane in your model ?

Ikeuchi : Yes, thank you for your question. The burst of star formation gives rise to the sequential explosions of supernovae, so that the superbubbles expand to the halo and the hot gas flows out. Meanwhile, this gas is cooled and returns to the disk plane after a radiative cooling phase. I believe that in our galaxy such a large scale circulation of gas through the halo occurs stationarily.

Nakamura : Did you consider the effect of a stellar component for the stability condition? I think that to check this effect is very important for your model.

Ikeuchi : No, I did not consider it. It is very important, I agree with you. 